\title{
Modeling Vehicle-to-Vehicle Line of Sight Channels and its Impact on Application-Layer Performance
}

\author{
Mate Boban*, Wantanee Viriyasitavat ${ }^{* \dagger}$, and Ozan Tonguz* \\ *Dept. of Electrical and Computer Engineering, Carnegie Mellon University, Pittsburgh, PA, 15213, USA \\ ${ }^{\dagger}$ Faculty of Information and Communication Technology, Mahidol University, Thailand \\ mboban@cmu.edu, wviriyas@andrew.cmu.edu, tonguz@ece.cmu.edu
}

\begin{abstract}
We analyze the properties of line of sight (LOS) channels in vehicle-to-vehicle $(\mathrm{V} 2 \mathrm{~V})$ communication. We use $\mathrm{V} 2 \mathrm{~V}$ measurements performed in open space, suburban, and urban environments. By separating LOS from non-LOS data, we show that a two-ray ground reflection path loss model with effective reflection coefficient range fits the LOS channels better than the frequently used free space path loss model. Two-ray model is a better fit not only in open space, but also in suburban and urban environments. We investigate the impact of using the modified two-ray model on the application-level performance metrics: packet delivery rate, throughput, latency, and jitter. Our results show that considerable differences arise in application performance when using the modified two-ray and free space models.
\end{abstract}

Categories and Subject Descriptors: C.2 [Computer Systems Organization]: Computer Communication Networks Keywords: Vehicle-to-Vehicle Communication, Line of Sight Channels, Two-ray Ground Reflection Model

\section{INTRODUCTION}

Enabled by the Dedicated Short Range Communication (DSRC) technology, vehicle-to-vehicle (V2V) communication is envisioned to support new cooperative Intelligent Transportation Systems (ITS) applications. To correctly simulate ITS applications, it is critical to utilize realistic channel models [1]. However, designing models that can be generalized to different environments is a difficult task. To that end, Karedal et al. in [2] perform measurements in rural, highway, suburban, and urban environments and conclude that the two-ray ground reflection model [3, Chap. 3.6] matched the path loss profile of the measurements in rural environment. In a similar study, Cheng et al. in [4] perform measurements in rural and highway environments and conclude that the two-ray model fits well with the measured path loss. In these studies, the measurement data was not separated into line of sight (LOS) and non-LOS. However, whenever the collected measurement data was predominantly of LOS type, the modified two-ray model fits the path loss well.

In this paper, we are interested in realistically modeling LOS V2V channels in different real-world environments. We start by analyzing the occurrence of LOS conditions on experimental datasets $[5,6]$. The videos recorded during the

Copyright is held by the author/owner(s). VANET'13, June 25, 2013, Taipei, Taiwan ACM 978-1-4503-2073-3/13/06.
Table 1: Measured proportion of LOS links.

\begin{tabular}{c|cccc}
\hline Environment & $\begin{array}{c}\text { Porto } \\
\text { Highway }\end{array}$ & $\begin{array}{c}\text { Pittsburgh } \\
\text { Suburban }\end{array}$ & $\begin{array}{c}\text { Porto } \\
\text { Urban }\end{array}$ & $\begin{array}{c}\text { Pittsburgh } \\
\text { Urban }\end{array}$ \\
\hline \% LOS links & $30 \%$ & $82 \%$ & $38 \%$ & $72 \%$ \\
\hline
\end{tabular}

experiments reveal that, while the proportion of LOS channels could change considerably depending on the environment and the time of day, LOS communication comprises a significant portion of the data (see Table 1).

To the best of our knowledge, this is the first study that: a) models the V2V LOS channels exclusively by separating LOS from non-LOS data using experiments performed in real-world environments; and b) assesses the impact of LOS channel model selection on throughput, packet delivery rate (PDR), latency, and jitter.

\section{MEASUREMENT DATASETS}

We used datasets collected in V2V measurement campaigns reported in [5] and [6]. The collected data and the videos of the experiments are freely available on the DRIVEIN project website ${ }^{1}$. The measurements, utilizing IEEE 802.11p (DSRC) radios, were performed in the following locations:

- Porto Open Space - $1 \mathrm{~km}$ route at approximate coordinates (lat,lon): 41.210615, -8.713418.

- Porto Urban - $9 \mathrm{~km}$ route at approximate coordinates (lat,lon): 41.153673, -8.609913.

- Pittsburgh Suburban - $7 \mathrm{~km}$ route at approximate coordinates (lat,lon): 40.4476089, -79.9398574.

The transmitting and receiving vehicles were driven in the same direction and in normal traffic conditions according to the traffic rules on the road. The trailing vehicle was equipped with a videocamera, which was later used to separate the collected data according to the observed LOS conditions. The measurements were performed between May 2010 and December 2011, all in mostly dry weather. Open Space and Urban experiments were performed in the afternoon hours ( 1 p.m. to 8 p.m.), whereas Suburban experiments were performed during nighttime (11 p.m. to 2 a.m.). Vehicles were equipped with a NEC LinkBird-MX V3, a development platform for vehicular communications, with omnidirectional antennas mounted on the roof of the vehicles. Packets were transmitted on the $20 \mathrm{MHz}$ channel at $6 \mathrm{Mbps}$ and transmit power of $10 \mathrm{dBm}$. Detailed information about

\footnotetext{
${ }^{1}$ http://drive-in.cmuportugal.org
} 


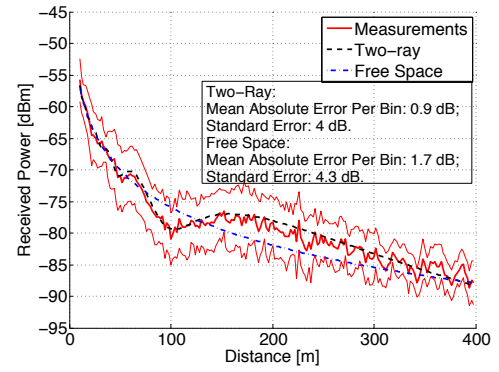

(a) Porto Open Space. Number of LOS data points: 61000. Average measured standard deviation: $3.3 \mathrm{~dB}$.

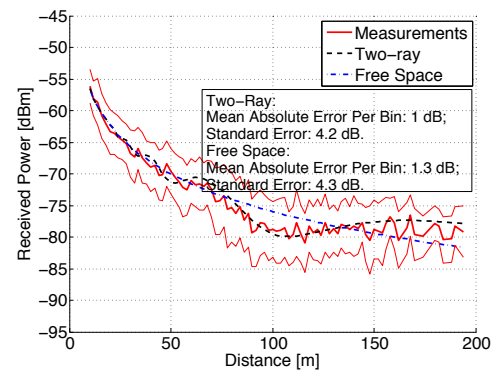

(b) Pittsburgh Suburban. Number of LOS data points: 11900. Average measured standard deviation: $4.1 \mathrm{~dB}$

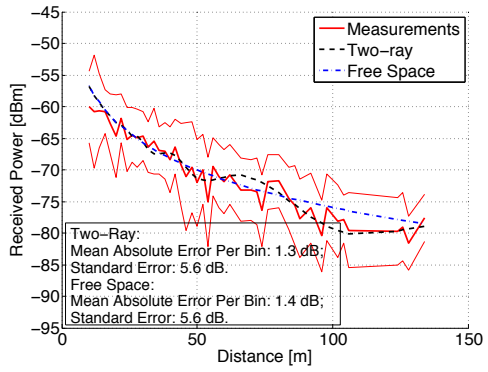

(c) Porto Urban. Number of data points: 4400. Average measured standard deviation: $5.3 \mathrm{~dB}$.

Figure 1: Comparison of received power collected during measurements with two-ray and free space models. Measurement data is placed in two meter distance bins. Only bins with more than 40 data points are included. Outer red lines represent one standard deviation around the mean received power for each bin. Results for additional environments and vehicular traffic densities are available in [7].

the devices and DSRC parameter setup can be found in [7], which also contains analysis of LOS channels in additional environments and with different vehicular traffic densities.

\section{MODELING THE V2V LOS CHANNELS}

Due to the inherent structure of the environment where most V2V communication occurs - over the face of road surface - the propagation characteristics of LOS communication are likely influenced by at least two dominant rays between transmitter and receiver: optical LOS ray and groundreflected ray. For this reason, we investigate in which environments and under which traffic conditions the two-ray ground reflection model fits the measurements well. The resultant electric field, $E_{T O T}$, of the electromagnetic wave at the receiver for the two-ray model can be calculated by accounting for line of sight and ground-reflected component as follows [3, Chap. 3.6, Eq. 3.39]:

$$
\begin{aligned}
E_{T O T} & =E_{L O S}+E_{\text {ground }} \\
& =\frac{E_{0} d_{0}}{d_{L O S}} \cos \left(\omega_{c}\left(t-\frac{d_{L O S}}{c}\right)\right) \\
& +R_{\text {ground }} \frac{E_{0} d_{0}}{d_{\text {ground }}} \cos \left(\omega_{c}\left(t-\frac{d_{\text {ground }}}{c}\right)\right),
\end{aligned}
$$

where $E_{L O S}$ and $E_{\text {ground }}$ are the electric field (E-field) of the LOS and ground-reflected ray, respectively, $E_{0}$ is the E-field at a reference distance $d_{0}$ (in the antenna far field), $\omega_{c}$ is the angular frequency, $t$ is the time at which the Efield is evaluated, $R_{\text {ground }}$ is the ground reflection coefficient, $d_{L O S}$ is LOS distance between antennas, $d_{\text {ground }}=$ $\sqrt{\left(h_{t}+h_{r}\right)^{2}+d^{2}}$ is the propagation distance of the groundreflected ray, where $h_{t}$ and $h_{r}$ is the height of the transmitting and receiving antenna, respectively, and $d$ is the ground distance between the antennas. When the originating medium is free space, $R_{\text {ground }}$ is calculated as follows for horizontal polarization [3, Chap. 3.6, eq. 3.25]:

$$
R_{\text {ground }}=\frac{\sin \theta_{i}-\sqrt{\epsilon_{r}-\cos ^{2} \theta_{i}}}{\sin \theta_{i}+\sqrt{\epsilon_{r}-\cos ^{2} \theta_{i}}}
$$

where $\theta_{i}$ is the incident angle, and $\epsilon_{r}$ is the relative permittivity of the material. On the other hand, the free space propagation model [3, Chap. 3.2] assumes the existence of the LOS component only, i.e., the first term in Eq. 1. The ensuing received power $P_{r}$ (in watts) is calculated as $P_{r}=\frac{\left|E_{T O T}\right|^{2} \lambda^{2}}{4 \pi \eta}$, where $\lambda$ is the wavelength and $\eta$ is the intrinsic impedance $(\eta=120 \pi$ ohms in free space).

The idealized two-ray model is an approximation of the actual V2V channel, since the reflection coefficient is affected by the antenna location, diffraction over the vehicle roof below antenna, and the roughness of the road, among other. For this reason, when calculating $R_{\text {ground, }}$, we model the relative permittivity $\epsilon_{r}$ to obtain the effective range of reflection coefficient values across different incidence angles. To estimate $\epsilon_{r}$, we used the Porto Open Space dataset, as it was the environment most resembling the theoretical conditions for the two-ray ground model - a flat, empty road with few reflectors. We performed curve fitting by minimizing the square residuals, which yielded $\epsilon_{r}$ value of 1.003 .

Figure 1 shows the received power measurements for LOS links in environments described in Section 2, compared with the two-ray and free space models. Not surprisingly, among the analyzed environments, Porto Open Space results in the best match between the measurements and the two-ray model (Fig. 1(a)). Results for Pittsburgh Suburban, where the experiments were performed during late night with almost no traffic, also show a good agreement with the tworay model (Fig. $1(\mathrm{~b})$ ). Figure. $1(\mathrm{c})$ shows that the two-ray model fits the LOS data in Porto Urban environment slightly better than the free space model. This result implies that the ground-reflected ray is still a significant factor in path loss modeling for LOS channels, despite the vehicles being surrounded by buildings that can create strong reflections.

\section{IMPACT OF CHANNEL MODEL ON APPLICATION-LEVEL PERFORMANCE}

We perform simulations using the NS-2.35 simulator to evaluate the impact of LOS channel model selection on packet delivery rate, throughput, latency, and jitter. A scenario with two vehicles is set up to assure that interference and routing protocol characteristics do not impact the communication. In the simulation, the overtaking assistant seethrough system [8] is used as an illustrative application. The vehicle in front continuously streams MPEG4 video [9] to the 

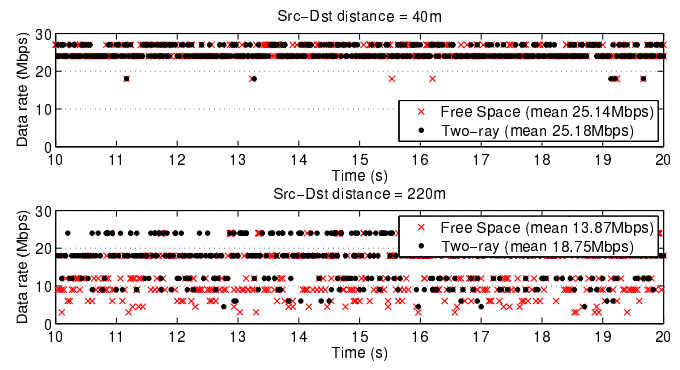

Figure 2: Instantaneous data rate used to transmit a packet for a given distance.

trailing vehicle over Real-time Transport Protocol (RTP). IEEE 802.11p standard is implemented at the Physical and MAC layer. To match the measurement settings, the transmit power (plus antenna gains) is set to $12 \mathrm{dBm}$ and $\epsilon_{r}$ is set to 1.003. The modified two-ray channel model and free space model are used as path loss models. According to the measurements in Porto Open Space environment, the variation around the mean power for LOS links - i.e., the small scale signal variation due to multipath - can be well modeled by a normal random variable. Therefore, atop the path loss, we add a normally distributed random variable with zero mean and standard deviation of $3.3 \mathrm{~dB}$. Receiver-based Auto Rate (RBAR) algorithm [10] is used as an adaptive modulation control scheme. RBAR adjusts the data rate based on the perceived signal-to-noise (SNR) value measured from the ACK packets. The SNR thresholds used in our simulations are based on measurements in [11].

Figure 2 shows the difference in terms of selected data rates over time when the two channel models are used. Observe that, when the source and destination vehicles are close to each other (e.g., $40 \mathrm{~m}$ apart), the SNR values are usually larger than $20 \mathrm{~dB}$ for both models. Thus, most packets are transmitted at the maximum rate of $27 \mathrm{Mbps}$. On the other hand, when the source-destination distance increases, the difference in the selected rate becomes more pronounced the second plot in Fig. 2 shows that the mean data rate varies by up to $5 \mathrm{Mbps}$ - from application standpoint, such disparity might mean a difference between a successful and a failed transmission.

Figure 3 shows the performance of the four applicationlevel metrics when the two channel models sure used. While the models generate similar results in terms of the amount of transferred data, the two-ray model results in 30\% decrease in terms of packet latency and jitter. This result emphasizes the importance of LOS channel model selection; the observed difference in latency and jitter would considerably change the perceived performance of real-time applications.

\section{CONCLUSIONS}

Based on $\mathrm{V} 2 \mathrm{~V}$ measurements performed in open space, suburban and urban environment, we establish that the modified two-ray ground reflection model is a better match for LOS channels than the often used free space model. The two-ray model matches the measurements particularly well when the number of surrounding objects that generate additional reflections (e.g., other vehicles or buildings) is low. Based on these findings, we perform simulations to explore the impact of LOS channel model selection. The results in-
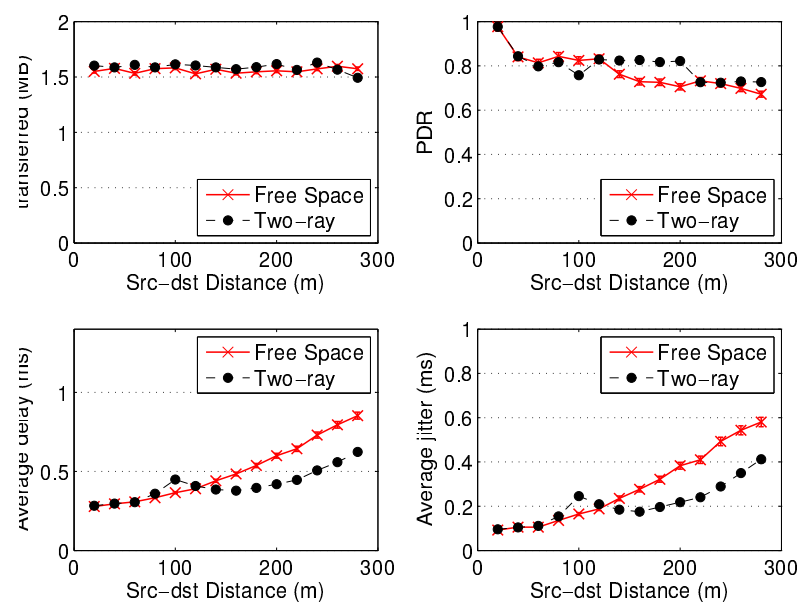

Figure 3: Application performances in terms of throughput, packet delivery rate, latency, and jitter when different LOS channels are used.

dicate considerable difference in terms of delay, jitter, and packet delivery rate when two models are used. This emphasizes the importance of using the correct channel model for LOS links. Since in terms of computational complexity the two channel models are equally simple to implement,we advocate the use of modified two-ray model for simulating V2V LOS channels.

\section{REFERENCES}

[1] C. F. Mecklenbrauker et al., "Vehicular Channel Characterization and its Implications for Wireless System Design and Performance," Proceedings of the IEEE, vol. 99, no. 7, pp. 1189-1212, 2011.

[2] J. Karedal et al., "Path Loss Modeling for Vehicle-to-Vehicle Communications," IEEE Transactions on Vehicular Technology, vol. 60, no. 1, pp. 323 -328, 2011.

[3] T. S. Rappaport, Wireless Communications: Principles and Practice. Prentice Hall, 1996.

[4] L. Cheng et al., "Doppler Spread and Coherence Time of Rural and Highway Vehicle-to-Vehicle Channels at 5.9 GHz," in IEEE GLOBECOM, 2008, pp. 1-6.

[5] R. Meireles et al., "Experimental Study on the Impact of Vehicular Obstructions in VANETs," in IEEE VNC, 2010, pp. 338-345.

[6] M. Boban et al., "Exploiting the Height of Vehicles in Vehicular Communication," in IEEE VNC, 2011.

[7] M. Boban et al., "Modeling Vehicle-to-Vehicle Line of Sight Channels and its Impact on Application-Level Performance Metrics," Carnegie Mellon University, T-SET - U.S. DOT University Transportation Center, Tech. Rep., 2013.

[8] P. Gomes et al., "The See-Through System: from Implementation to Test-drive," in IEEE VNC, 2012.

[9] A. Matrawy et al., "MPEG4 Traffic Modeling Using the Transform Expand Sample Methodology," in IEEE Workshop on Networked Appliances, 2002, pp. 249-256.

[10] G. Holland et al., "A Rate-adaptive MAC Protocol for Multi-hop Wireless Networks," in ACM conference on Mobile computing and networking, 2001, pp. 236-251.

[11] A. Paier et al., "Performance Evaluation of IEEE 802.11p Physical Layer Infrastructure-to-Vehicle Real-World Measurements," in Proceedings of ISABEL, November 2010. 